\title{
A Production/Transaction-Related Model Using Control Theory
}

Daisuke Doyo, Aoyama Gakuin University, Japan

Katsuhiro Sakamoto, Aoyama Gakuin University, Japan

Katsuya Aoki, Mitsubishi Motors Corporation, Japan

\begin{abstract}
In recent years, the need for wide-ranging kaizen/improvements has arisen in relationships with suppliers and other transaction partners in response to rising demands, including increases in profit and reductions in time. A method that results in such improvements is the application of a servo-mechanism control to manage inventory ordering. It is believed that if control theory is applied to the overall supply chain, it may enable an optimization of the supply chain, which fits the needs of modern society. This study proposes a prototype of the supply chain model of contemporary society, which applies the control theory and evaluates the validity of this model.
\end{abstract}

Keywords: Supply Chain Management, Supply Chain Design

\section{INTRODUCTION}

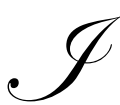

n today's society, when an enterprise faces the need to reduce inventory and shorten supply time, there are many factors that cannot be controlled within the enterprise. It is thus necessary to consider the company's relationships with other enterprises. A factor that should be considered is "transactions," such as comprehensive win-win relationships that include suppliers and vendors. Applying servo-mechanism theory (control theory) to inventory management, H. A. Simon conducted research on the control of order quantity through a cycle that takes into account the difference between planned quantities and actual values and incorporates them into subsequent draft plans. The approach described in his article is one method currently used in control systems to keep control variables constant.

Recently, the transaction environment is such that an enterprise cannot, by itself, exert enough control to yield results according to forecasts; thus, there is an increasing use of supply chain management that offers a scheme for overall optimization (Najafi, Bennett, 1984, Wiendahl, 2001, Ortega, Lin, 2004, Markus, Peter, 2006). In such an environment, when an enterprise wants to appropriately manage its quantity of inventory and volume of production, it is crucial to aim at wide-ranging controls extending to transactions influenced strongly by relationships with other enterprises. It is worth considering Simon's model, which is well-adapted to modern society as a spreading approach to supply chain management. As such, it is an approach whose scope is applicable to transactions with other enterprises, not merely production within one enterprise.

This research aims at practical application of a model that extends the scope of control theory to cover production and transactional relationships. It also aims at evaluating the validity of the new model by first designing a prototype, and then looking at both the influence over the supplier provided by control on the manufacturer's side and that over the manufacturer provided by control on the supplier's side.

\section{CONTROL THEORY}

The main, distinctive characteristic of control theory is its scheme of approximating set target values, while providing feedback consisting of previously output result values to new input points. This makes it suitable for a loop structure, such as a Plan-Do-See cycle. It is believed that if control theory is applied to production control, so as to automatically control plant production rates and quantities of product and parts inventories, it would lead the way to the control of numerical values, such as planned production values, quantities of parts inventories, and the quantity of products produced. Doing so would thus result in fewer over- and underruns of various numerical values. Moreover, this would provide a distinctive characteristic of control; namely, the equivalent conversion of transfer functions. By taking advantage of this characteristic, it would be possible to alter the intricate details of models in parts, applying great detail to parts that are strongly affected and with only coarse detail to parts that are weakly affected. It would thus be possible to derive results with the same precision without the need for excessive calculations.

Moreover, it would be possible to treat control models as mathematical models. When mathematical models would be input into a computer, it would be possible to use them as such in simulations, predictions, and optimizations. 


\section{MODEL DESIGN}

\subsection{SCM Model}

Production is the focus of manufacturers, and as such, it usually requires the most number of processes. The external contact points where transactions take place are the focus of model design because they are the crucial points at which matters are expected by both sides to proceed smoothly. In addition, model design incorporates the occurrences of a number of problems. These problems may not originate from a single enterprise; the problems that arise among the enterprise's transaction partners could have a scope that affects the enterprise itself. Such problems may include inventory oversupply due to problems on the manufacturer's production, inventory shortages due to problems in the suppliers' product lines, as well as cases in which an enterprise cannot place orders with a supplier because the supplier has already exceeded its production capacity with orders from other companies. This research attempts to construct a simple supply chain centered on manufacturers and identifies the flow of goods and information by applying control theory.

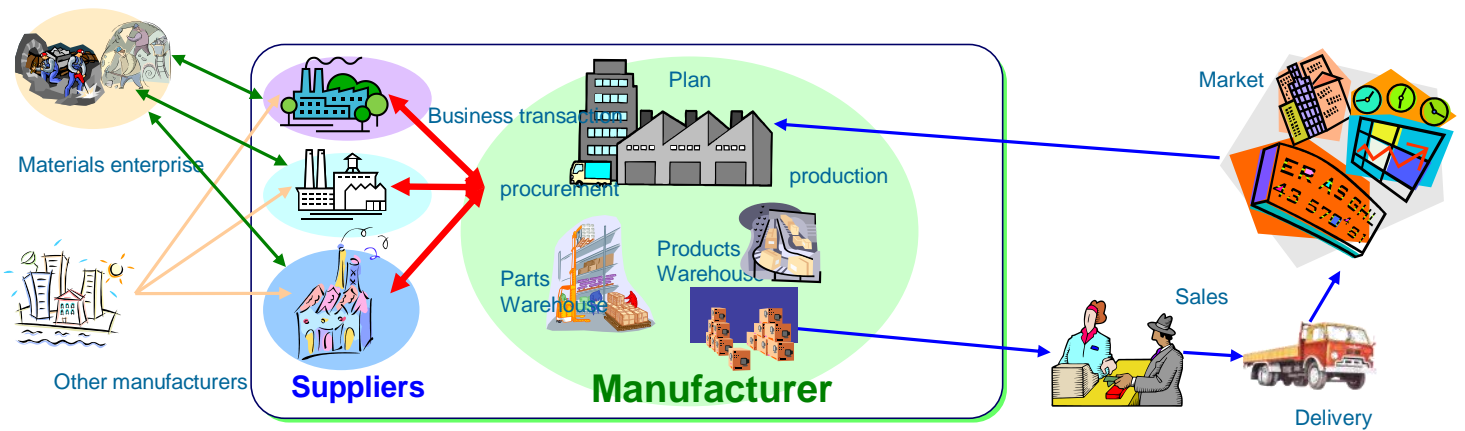

Fig. 1.Domain of the model

\subsection{Model Outline}

As shown in Figure 1, the domain of our model's design focuses on transactions between manufacturers and suppliers from the viewpoint of the manufacturer. The transaction units of an enterprise consist of five divisions: product production, product warehousing, parts warehousing, parts procurement, and production planning. Only one manufacturer is modeled here; however, other manufacturers exist whose functions affect only the production line operations of suppliers. In addition, product manufacturers and parts suppliers together have adopted the min-max system with the market production method.

Figure 2 shows the manufacturer and suppliers in a format that applies control theory. The input to the model is the volume of production that the market demands; the output is the quantity of the product in the market. The functions of the manufacturer and suppliers are controlled in order to minimize the differences between input and output formats.

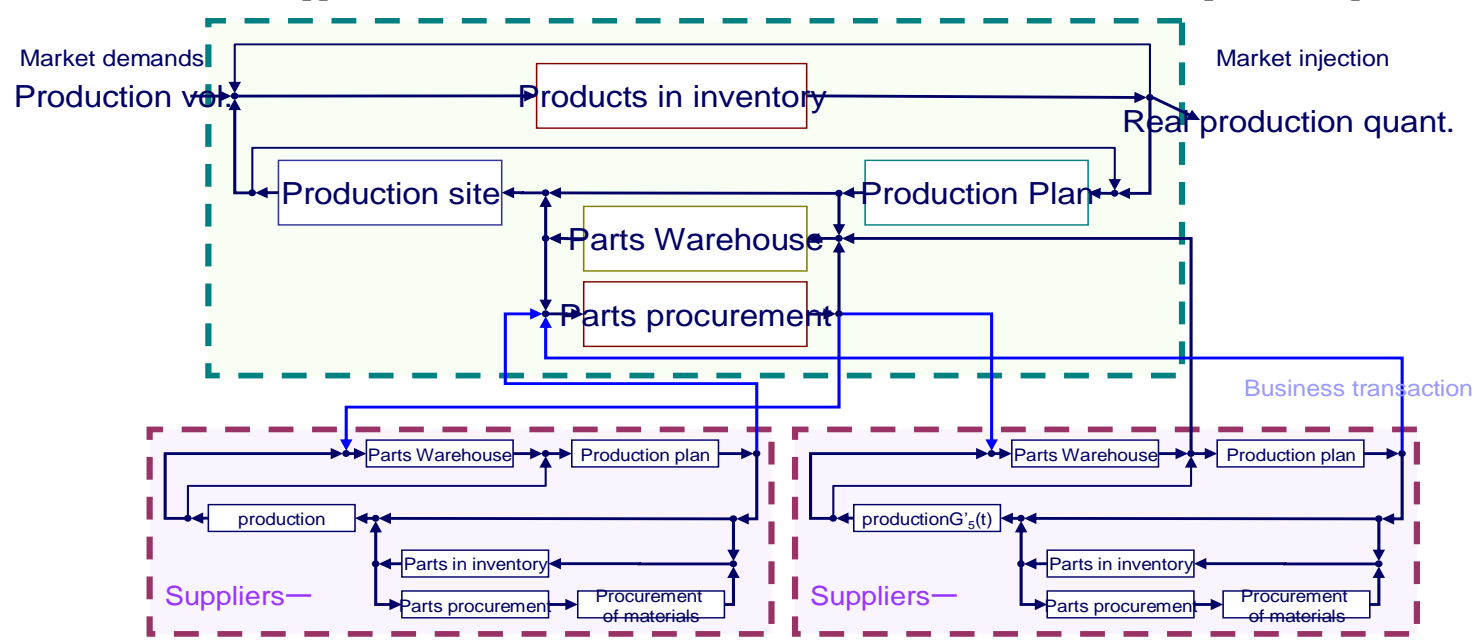

Fig. 2.Outline of model 


\subsection{Detail of Model}

More detailed versions of Figure 2 are shown in Figure 3, which covers the manufacturer, and Figure 4, which covers the suppliers.

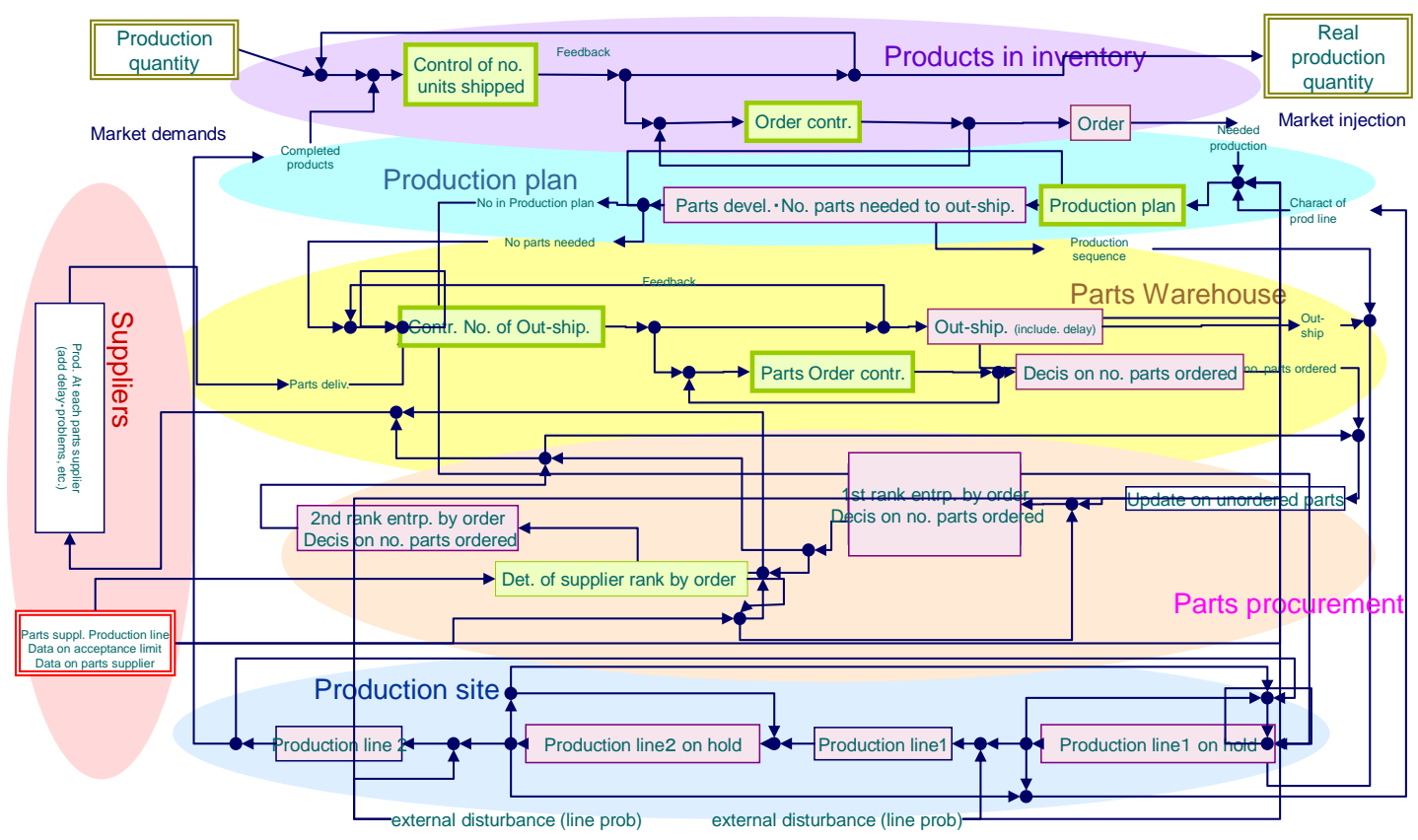

Fig. 3.Model of manufacture

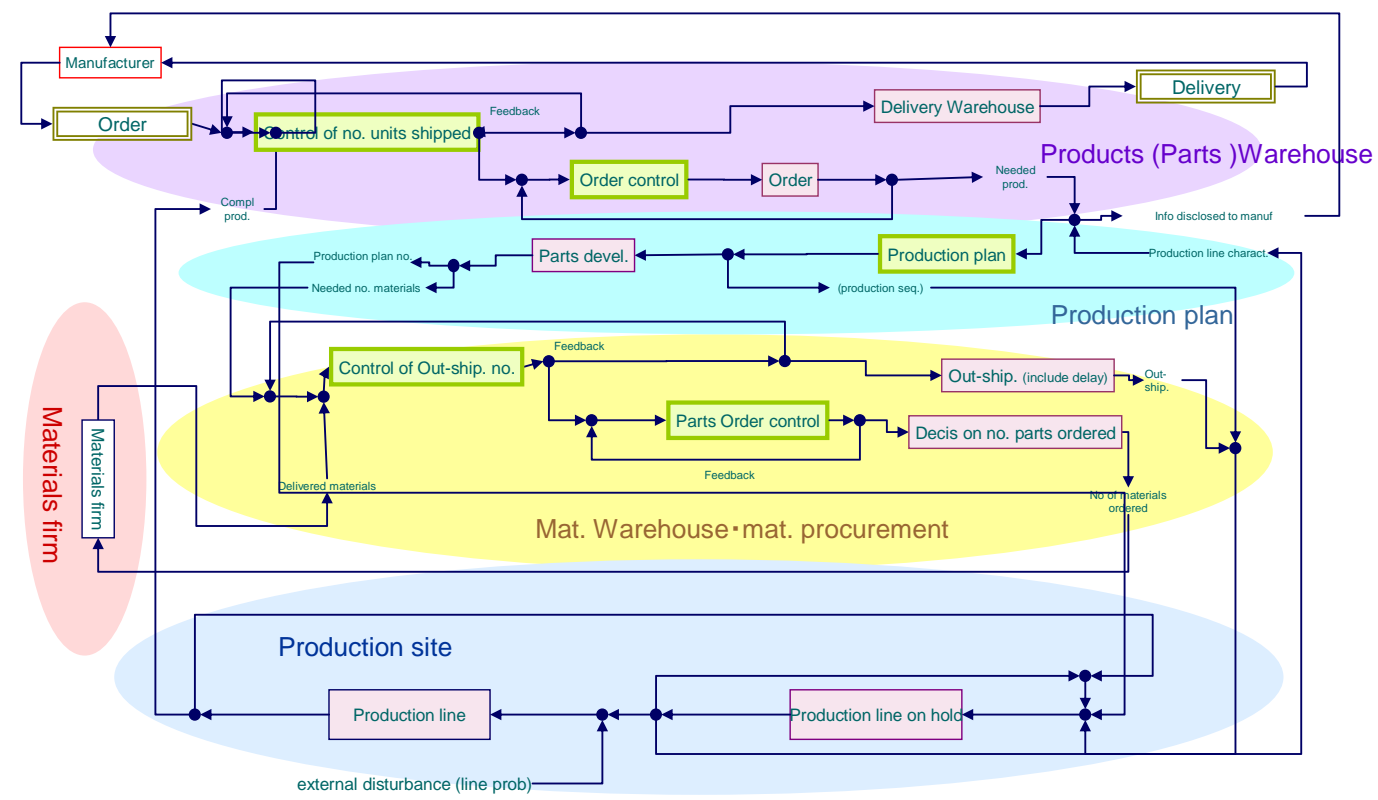

Fig. 4.Model of suppliers 
The information used when a manufacturer decides upon the suppliers to place orders consisting of the order results (total quantity of orders), previous experience regarding postponements of the scheduled dates of delivery (total postponement time/total order quantity), the production capacity of the suppliers (no. of units produced/time), suppliers' product quality, and product prices. An additional set of included information consists of the setting of indices reflecting the receptiveness of the supplier to orders and the relationship between manufacturer and suppliers. This data is weighted and orders are placed with suppliers that have high evaluations. It is possible to make a model that reflects the attitude of the suppliers quite well by weighting their receptiveness to orders. As for the process of determining order quantity, the resulting scheme channels orders from the manufacturer to high-priority suppliers, as reflected in the coordination between the manufacturer and the suppliers regarding the number of units produced and the date of delivery.

\subsection{Controls}

Several individual controls are worth mentioning. First, the controls on the number of inventory and order sources, which are used to stabilize the quantity of inventory within a prescribed scope, are explained. The quantity of inventory is stabilized within the target scope by reducing the ordering point if the quantity of inventory has exceeded the UCL (Upper Control Limit) within the prescribed scope, and by increasing the ordering point if the quantity of inventory has exceeded the LCL (Lower Control Limit)within the prescribed scope. Next, controls to alter production capacity so as not to exceed the number in the production plan are considered.

In this case, when the numbers needed for production plans have continued to exceed a certain value, the processing capacity is boosted by increasing the basic production capacity. For example, if due to some accident, the production line is halted and the number of unproduced units temporarily rises excessively, the situation is controlled by raising production capacity after the production line is restored and then returning production to its original value after stability has been restored.

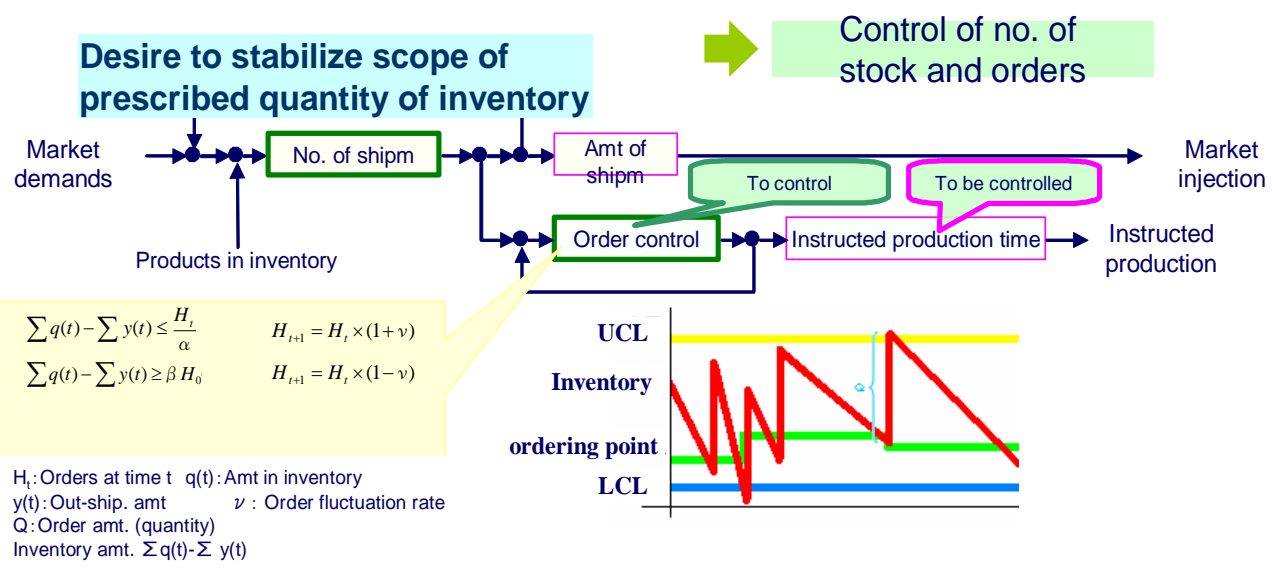

Fig. 5.Control of orders

\section{SIMULATION RESULT}

The validity of the model in situations of total operation is checked. In one environment, the model operated without any particular external disturbance, and its production capacity control function was observed. This control function stabilizes production capacity by altering it in accordance with input formulas. Under the formulas and environments that were input to check the validity, the model immediately ran out of inventory, assuming an initial production capacity of 50 units. Subsequently, fluctuations stopped and stability was restored when the value of production capacity was controlled after being raised to 70 units. As for the quantity of inventory, there were no shortages of inventory after the production capacity was adjusted. The results derived here can be used as references for initial settings when establishing production plans. 


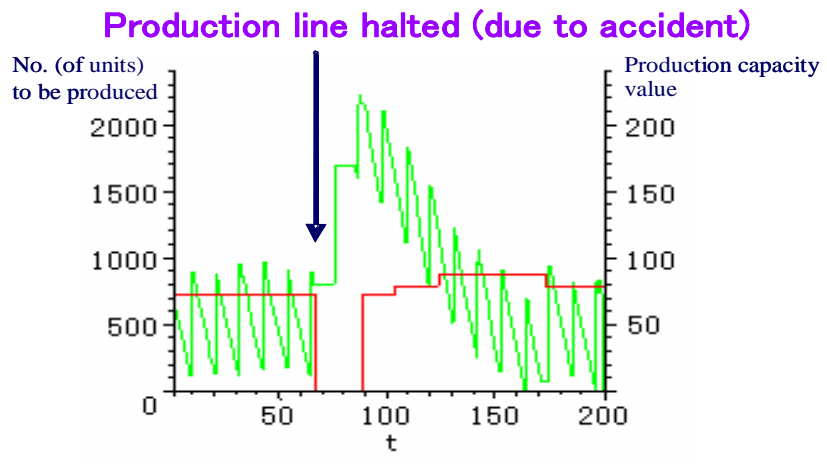

Fig. 6.Simulation of control of production capacity

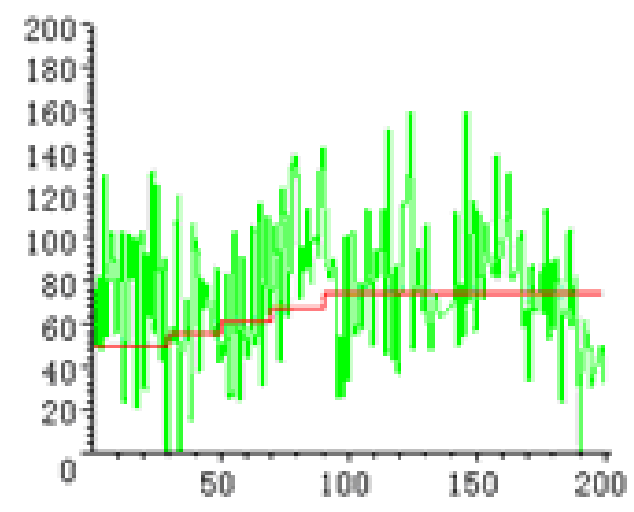

Fig. 7. Market requirements

Next, an accident was generated in the manufacturer's production line and the line was halted for some time. When the stoppage was five hours long, stability was restored immediately; however, the number of units in inventory dropped slightly. Moreover, there was no effect on other sections besides production.

When the stoppage lasted for ten hours, the stock in inventory was restabilized after some time; the manufacturer's production line restarted before the parts warehouse on the manufacturer's side, and the delivery warehouse on the supplier's side had filled up. The supplier's production line was not halted either.

In the case of a 20-hour stoppage, the supplier's production line stopped because the manufacturer's parts warehouse and the supplier's delivery warehouse were completely filled. Because the production line of the supplier stopped and the supplier faced the resulting increases in orders to be filled from other manufacturers, the quantity of supplied parts was not readily restored and the number of the inventory of finished goods was not stabilized, even after the production line of the manufacturer in the model was restarted.

These results indicate that a stoppage has no effects, in particular, if the line can be restored within five hours. The line situation can also be restored to its preaccident status in the case of a 10-hour stoppage. However, in the case of a 20-hour stoppage, there is an effect on the supplier's side. Consequently, the results show potential for a considerable loss unless the line can be restarted within approximately ten hours at the most. 


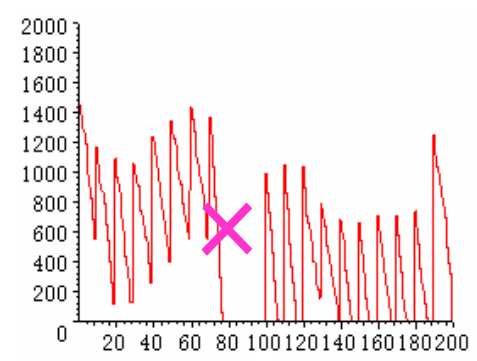

Fig. 8.Stock in inventory

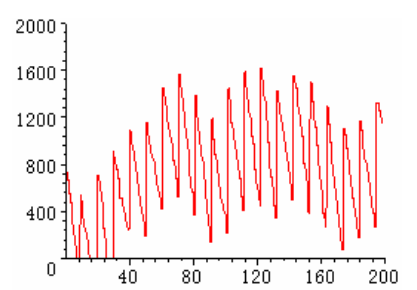

Fig. 9.Products in inventory in the case of a 5-hour stoppage

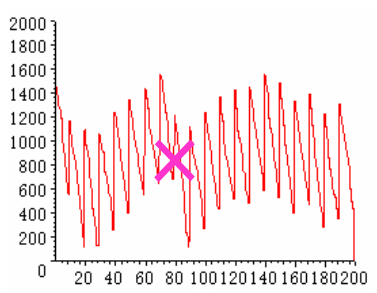

Fig. 10.Products in inventory in a 10-hour stoppage

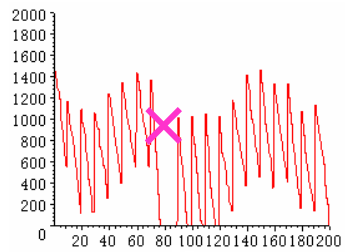

Fig. 11.Products in inventory in a 20-hour stoppage

This study has proposed a model that applies control theory as a production control model and a transaction model. The distinctive characteristic of this study is the modeling of transaction controls. However, a certain amount of decision-making must take place in a format that makes control models applicable to actual transaction negotiations. The control model in this study is only capable of accommodating decision-making by the model user in the initial input stage. Consequently, some preconditions were made on the transaction model. This format allows for the determination of how well the numerical values approximate the objectives when the relationships with suppliers move ahead in patterns of different conditions set by the controller.

These conditions were to maintain the quality and prices of parts provided by suppliers so as to allow the operating rate of the production line to be influenced as needed by the transactions. By doing so, the controller's 
assessment may change, depending on whether the situation allows for an emphasis on price and quality or the situation demands an emphasis on speed above all. Such changes in assessment may also be chosen according to numerical assessments, including the occurrence of losses. Thus, a difference between this and other simulation models is that in this model, all transaction-related decision-making may be performed numerically.

In addition, calculations to determine the final results are by composite mathematical expressions based on the transaction unit. It may take some time to compose expressions; however, conventional simulations can assess whether the design of the simulation model is applicable to operations in the same categories. Moreover, if the composite expressions are again disaggregated (returned to their status before the composition of expressions) and different input formulas are substituted, it is possible to view the trends of values according to the individual composite expressions. If inverse Laplace transforms are applied after having composed feature expressions for each transaction unit subject to Laplace transforms, one composite formula that represents multiple expressions is obtained. To perform this task as a normal simulation, we proceed in two phases, with the results of Expression A inserted into the processing of Expression B. Then, there is the possibility of instances requiring the redesign of models that include both expressions.

As a result of the simulation in this research, it seems possible to extend the application of Simon's control of inventories and order quantity by servo-mechanism theory to cover production volume and transaction processes.

Moreover, it is possible to link this to a reduction in problem-resolution processes in the Plan-Do-See cycle through repetition of the PDS cycle, even though complete automation of all processes (which would require flawless results) was not achieved. This may be regarded as a model that allows automatic alteration (=automatic control) of the setting values to accommodate changes, depending on the status. When conventional simulation software is used, the user must learn how to operate it in accordance with the software's peculiar characteristics. However, mathematical software has a wide range of other applications and can be applied in other simulations. Thus, it could reduce learning time.

Models using control theory can acquire numerical values to stabilize the volume of production and quantity of inventory, as well as critical points to restabilize the system against external disturbances. They can also play a supporting role in plan proposals using the acquired data to make production plans that take into account the supplier. To incorporate the scheme of this model into a real social setting and enhance the model's precision, addition of model elements adapted to the scheme of the production worksite is required.

\section{AGENDA FOR FUTURE RESEARCH}

In some views, control engineering itself is already in a period of maturity. However, it cannot cope well enough with complex, nonlinear problems, but can cope with problems that are easy to resolve.

The real world contains vaguenesses and complexities that cannot be measured physically by building mathematical models based on elimination and abstraction. It is difficult to approximate such matters and demonstrate the models' consistency with the real world. This research is an attempt to build a model that incorporates elements of control engineering to cover nonlinear supply chain management. However, it is only the first step toward the complete modeling of all nonlinear supply chains, corporate transactions, and market trends.

It is better to think of this research in terms of the question, "Is control theory useful as one technique to control supply chain management?" rather than "Does the scope of control theory also cover supply chain management?" This research does not apply the complete set of control theory. It is not applicable everywhere in the same format, and requires alterations according to conditions and usage requirements. Control theory may be regarded as one format of the alterations, and control theory for chemical, mechanical, and electrical applications is not applicable directly to supply chain management. However, "control of the supply chain" may well be established as one transactional model in the future.

\section{AUTHOR INFORMATION}

Dr. Daisuke Doyo is an Assistant Professor in the School of Science and Engineering at Aoyama Gakuin University, Japan. He received his Ph.D. degree in Keio University in 2008. His current study interests are in the Skill Transfer Management and Supply Chain Design.

Dr. Katsuhiro Sakamoto is an Associate Professor in the School of Science and Engineering at Aoyama Gakuin University, Japan. He received his Ph.D. degree in Aoyama Gakuin. His current study interests are in the Supply Chain Management.

Katsuya Aoki is working for Mitsubishi Motors Corporation and doing the business related to the supply chain management. 


\section{REFERENCES}

1. Najafi H, Bennett J E.: Inventory-production optimization using optimal control theory techniques. Proc Annu Southeast Symp Syst Theory, Vol. 16 (1984), 12-15.

2. Ortega M, Lin L: Control theory applications to the production-inventory problem: a review. Int J Prod

ResVol.42(2004), No.11, 2303-2322.

3. Simon, H A: On the application of servo-mechanism theory in the study of production control. Econometrica, Vol. 20 (1952) 247-268.

4. Schwaninger M, Vrhovec P: Supply system dynamics: distributed control in supply chains and networks. Cybern Syst, Vol.37 (2006), No.5, 375-415.

5. Wiendahl H-P. : Backlog-Oriented Automatic Production Control. CIRP Ann, Vol. 50 (2001), No.1, 331-334. 